\title{
Supplement Information to Effects of Solvent Viscosity on Ligand Interconvesrsion Dynamics in the Primary Docking Site of Heme Proteins
}

\author{
Seongheun Kim, Jeonghee Heo, and Manho Lim* \\ Department of Chemistry, Pusan National University, Busan 609-735 Korea \\ RECEIVED DATE (automatically inserted by publisher); mhlim@pusan.ac.kr
}

\section{Experimental methods}

The details of the time-resolved mid-IR spectrometer used here are described elsewhere. ${ }^{1}$ Briefly, two identical home-built optical parametric amplifiers (OPA), pumped by a commercial Ti:sapphire amplifier, are used to generate a visible pump pulse and a mid-IR probe pulse. Pump pulse at $580 \mathrm{~nm}$ with $3 \mu \mathrm{J}$ of energy was generated by frequency doubling of a signal pulse of one OPA. Tunable mid-IR probe pulse was generated by difference frequency mixing of the signal and idler pulse of the other OPA. The polarization of the pump pulse was set at the magic angle (54.7) relative to the probe pulse to recover the isotropic absorption spectrum. The broadband transmitted probe pulse is detected with a 64-elements $\mathrm{N}_{2}(l)$-cooled HgCdTe array detector. The array detector is mounted in the focal plane of a 320 $\mathrm{mm}$ monochromator with a $150 \mathrm{l} / \mathrm{mm}$ grating, resulting in a spectral resolution of ca. $1.4 \mathrm{~cm}^{-1} /$ pixel at $2100 \mathrm{~cm}^{-1}$. The signals from each of the detector elements were amplified with a homebuilt 64-channel amplifier and digitized by a 12-bit ADC. Chopping the pump pulse at half the repetition frequency of the laser and computing the difference between the pumped and unpumped absorbance determine the pump-induced change in the absorbance of the sample $\Delta A$. Due to the excellent short-term stability of the IR light source $(<0.5 \% \mathrm{rms})$, less than $1 \times 10^{-4} \mathrm{rms}$ in absorbance units after $0.5 \mathrm{sec}$ of signal averaging is routinely obtained without single shot referencing with an independent detector. The pump spot was made larger than the probe spot to ensure spatially uniform photoexcitation across the spatial dimensions of the probe pulse. The instrument response function is typically $150 \mathrm{fs}$.

Samples in glycerol/water mixtures were prepared by dissolving $11 \mathrm{mM}$ lyophilized skeletal horse Mb (Sigma) in either $65 \%$ or $75 \%$ by weight glycerol- $\mathrm{D}_{2} \mathrm{O}$ buffered with $0.1 \mathrm{M}$ potassium phosphate (pD 7.4). The solution was equilibrated with $1 \mathrm{~atm}$ of ${ }^{13} \mathrm{CO}$ (Aldrich, $99.3 \%{ }^{13} \mathrm{C},<1.8 \%,{ }^{18} \mathrm{O}$ ) and reduced with ca. 2 equivalent concentration of freshly prepared sodium dithionate (Aldrich). The reduced $\mathrm{Mb}$ was stirred under $1 \mathrm{~atm}$ ${ }^{13} \mathrm{CO}$ for at least 1 hour. To remove light scattering sources such as dust particles and denatured protein aggregates, samples were filtered through a $5-\mu \mathrm{m}$ membrane filter before loading in a gastight $50-\mu \mathrm{m}$-pathlength sample cell with $\mathrm{CaF}_{2}$ windows. The trehalose sample was prepared by combining equal amounts of an $11 \mathrm{mM} \mathrm{Mb}{ }^{13} \mathrm{CO}$ with a $1.5 \mathrm{M}$ trehalose solution. Both solutions were prepared in $\mathrm{D}_{2} \mathrm{O}$ buffered with $0.1 \mathrm{M}$ potassium phosphate. A $0.2 \mathrm{ml}$ aliquot of the resulting solution was layered on a $\mathrm{CaF}_{2}$ window and dried for $\sim 4 \mathrm{~h}$ under a $\mathrm{CO}$ atmosphere in a desiccator. The sample was then sealed by applying vacuum grease to the circumference of the window and pushing another $\mathrm{CaF}_{2}$ window against the first one. The residual water, estimated from vibrational spectra of trehalose (band at $2936 \mathrm{~cm}^{-1}$ ) and $\mathrm{D}_{2} \mathrm{O}$ (band at $2504 \mathrm{~cm}^{-1}$ ), was $\sim 1.3 \mathrm{D}_{2} \mathrm{O}$ molecules per trehalose molecule ( $\sim$ \% by weight).
During data collection the sample cell was rotated sufficiently fast so that each pump pulse illuminated a fresh volume of the sample. The temperature of the rotating sample cell was maintained at $283 \pm 1 \mathrm{~K}$. The integrity and concentration of sample was checked using UV-Vis and FT-IR spectroscopy.

Acknowledgement. This work was supported by the SRC program of MOST/KOSEF (R11-2000-070-04004-0) and the KRF Grant funded by the Korean Government (MOEHRD) (KRF-2005-070-C00063).

\section{References}

1. Kim, S.; Jin, G.; Lim, M., J. Phys. Chem. B 2004, 108, (52), 20366-20375.

2. Kim, S.; Heo, J.; Lim, M., Bull. Kor. Chem. Soc. 2005, 26, 151-156.

3. Kim, S.; Lim, M., J. Am. Chem. Soc. 2005, 127, (16), 5786-5787. 
